Article

\title{
Synthesis of Diblock Polyampholyte PAMPS-b-PMAPTAC and Its Adsorption on Bentonite
}

\author{
Ling Lin ${ }^{1,2, *}$, Yuanhao Luo ${ }^{3}$ and $\mathrm{Xin} \mathrm{Li}^{4}$ \\ 1 School of Chemistry and Chemical Engineering, Southwest Petroleum University, Chengdu 610500, China \\ 2 Oil \& Gas Field Applied Chemistry Key Laboratory of Sichuan Province (Southwest Petroleum University), \\ Chengdu 610500, China \\ 3 CCDC Drilling Fluid Technology Service Company, Chengdu 610051, China; haolyeureka@163.com \\ 4 Petroleum Engineering School, Southwest Petroleum University, Chengdu 610500, China; \\ up96157@gmail.com \\ * Correspondence: cowbolinling@swpu.edu.cn; Tel.: +86-28-83037306
}

Received: 27 November 2018; Accepted: 26 December 2018; Published: 30 December 2018

check for updates

\begin{abstract}
To study the adsorption of polyampholyte on bentonite (Bent), a block polyampholyte, PAMPS-b-PMAPTAC, comprised of 2-Acrylamido-2-Methylpropane Sulfonic Acid (AMPS) units and Methacrylamido Propyl Trimethyl Ammonium Chloride (MAPTAC) units, was synthesized using reversible addition-fragmentation chain transfer polymerization (RAFT) method. The block polyampholyte samples were characterized by FTIR, ${ }^{1} \mathrm{H}$ NMR and Gel Permeation Chromatography (GPC). The microstructure of block polyampholyte and random polyampholyte in deionized water indicated that uneven distribution of charged groups increased the entanglement of polymer chains. Addition of salt weakened the electrostatic interactions among charged groups, and, therefore, increased the zeta potential of polyampholyte in aqueous solutions. The adsorptive behaviors of PAMPS-b-PMAPTAC on Bent were studied using elemental analysis, and the effects of external factors were considered. The adsorption equilibrium of polymers on Bent was reached after $12 \mathrm{~h}$. Increased temperature and increased salinity exerted a positive and negative effect on the adsorption of polyampholyte, respectively. The molecular weight played as the decisive factor for the adsorption of polyampholyte in the absence of $\mathrm{NaCl}$, while the content of cationic groups acted as the main factor in the presence of $\mathrm{NaCl}$. Block polyampholyte exhibited higher adsorption than random polyampholyte in the absence of salt. XRD results also indicated that block polyampholyte had a better intercalation effect than random polyampholyte.
\end{abstract}

Keywords: block polyampholyte; bentonite; adsorption; AMPS; MAPTAC

\section{Introduction}

Polyampholyte is one kind of polymers with both cationic groups and anionic groups distributed on the same backbone. In aqueous solutions, these two kinds of charged groups in polyampholyte deionize, and the chains carry opposite charges. Driven by the electrostatic interaction and Van der Waal's force, polyampholyte chains assume various conformations, namely pole regime, pancake regime, and fence regime, under different conditions [1]. The conformation and behavior of the polymers in aqueous solutions, therefore, rely primarily on the monomer nature, charge asymmetry, charge distribution and chain length.

In terms of the monomer unit distribution in the backbone, polyampholyte can be classified as random polyampholyte with charged groups distributed statistically along the chain, alternating polyampholyte, and block polyampholyte with ionized monomer units bearing like charge located 
in different regions. In the laboratory, random polyampholyte is often synthesized by two methods. The first method is to add cationic monomers and anionic monomers, sometimes with other monomers, into one flask containing an aqueous solvent, and then initiate the polymerization. A number of reported random amphoteric polymers were produced in this one-pot approach [2,3]. The second method is to synthesize a cationic copolymer comprising cationic moieties and allyl units, followed by a hydrolyzation using $\mathrm{NaOH}$ or $\mathrm{HCl}$ to transform the allyl units to negatively-charged carboxyl units [3-5]. Using the two-step approach, You et al. obtained a cellulose-based polyampholyte by introducing both quaternized ammonium groups and a block of acrylic acid units via grafting [6].

Block polyampholyte is a class of responsive polymer attracting growing attention. The architecture of block polyampholyte includes AB diblocks [7,8], ABA triblocks [5,9-11], and other multi-blocks [12]. In the presence of weak acidic groups or weak basic groups along the backbone, block polyampholyte can change the net charge sign between positive and negative upon $\mathrm{pH}$ variation. Rich behaviors of block polyampholyte can be observed when external stimuli such as $\mathrm{pH}$ variation and temperature change is triggered. The dynamic protonation-deprotonation equilibrium of weak acid blocks (e.g., AA, stands for acrylic acid) and tertiary ammonium moieties (e.g., P2VP, stands for poly(2-vinyl-pyridine)) upon $\mathrm{pH}$ change endows the block polyampholyte with $\mathrm{pH}$-responsive behavior. Such behaviors can be found in the presence of polyacrylic acid (PAA) block and tertiary ammonium group block [7], or PAA block and P2VP block [13,14]. In these cases, at low $\mathrm{pH}$, both PAA block and P2VP block were protonated, and only the later was ionized and the polymer chain exhibited the same behavior as polycation; at intermediate $\mathrm{pH}$, deprotonated AA groups and protonated 2VP groups attracted each other. If the charge asymmetry was not significant, e.g. equimolar addition of anionic and cationic groups, the polymer chain tended to collapse into globular conformation and precipitate from the solution, as a result of intensified attractions between oppositely charged moieties [10]. When $\mathrm{pH}$ increased to over 8, both AA groups and 2VP groups underwent a process of deprotonation, and the polymer chain carried net negative charges and exhibited a behavior of polyanion. Dyakonova et al. argued that the contribution of electrostatic interactions includes Coulombic attraction and entropy gain through counterions release [10]. If hydrophobic association is introduced along with Coulombic interaction into the inter- and intra- chain interaction, polyampholyte may form a $\mathrm{pH}$-responsive and temperature-responsive hydrogel $[5,15]$.

Both random polyampholyte and block polyampholyte were applied in the area of drug delivery, tissue engineering, membrane, water treatment, and so on [16,17]. Mishra et al. delivered indomethacin using a random polyampholyte, poly(methacrylamido propyl trimethyl ammonium chloride/methacrylic acid), where the polyampholyte increased the release of indomethacin [18]. A random polyampholyte hydrogel consisting of [2-(methacryloyloxy)ethyl]-trimethylammonium chloride and 3-sulfopropyl methacrylate potassium salt had adjustable mechanical properties without affecting the non-fouling properties [19]. A membrane containing both sulfonic groups and quaternary ammonium groups exhibited a high selective separation of bovine serum albumin and lysozyme [20]. Copello et al. reported a random polyampholyte bearing carboxylate and 2-methylimidazole groups which can remove $\mathrm{Pb}$ (II) and $\mathrm{Cd}$ (II) from aqueous solution via adsorption [17]. Drug delivery requires polyampholyte to exhibit a conformational response to the external stimuli such as $\mathrm{pH}$ change, but other applications of polyampholyte rely on its strong interaction, especially adsorption, with substances such as proteins, foulants, metallic ions, and the like. The adsorption of random polyampholyte on a variety of absorbents was studied [17-20], while the adsorbents studied in the adsorption test of block polyampholyte are mainly silicon substrate [7]. The adsorption of block polyampholyte on other adsorbents, e.g., bentonite (Bent), was rarely reported.

Bent is a special kind of adsorbent carrying both high-density excessive negative charges on its flat layer surface and positive charges at its edges in aqueous solutions. The adsorption of polyampholyte bearing net negative charge on Bent acts as a decisive factor for the properties of water-based drilling fluid, as the adsorbed polyampholyte with excessive negative charges provides Bent with entropic protection against salt intrusion and heating. The adsorption of random polyampholyte on Bent has 
been reported in a previous paper [21]. The molecular composition and intrinsic viscosity of random polyampholyte exerted different effects on the adsorption of random polyampholyte. However, to the best of our knowledge, the adsorption of block polyampholyte on Bent and similar adsorbents has rarely been discussed, as most of the polymers interacted with Bent studied in previous papers can be classified as the polycation or polyanion based on polyacrylamide [22-24].

The main difference between random polyampholyte and block polyampholyte lies in their monomer sequence distribution. To study the effect of monomer sequence, charge asymmetry and molecular weight on the adsorption of polyampholyte on Bent, the authors availed themselves of the reversible addition fragmentation transfer polymerization (RAFT) method to synthesize both random polyampholyte via a one-step reaction and block polyampholyte with net negative charges via a two-step reaction, and then conducted the adsorption experiments to compare the adsorptive ability between them.

\section{Materials and Methods}

\subsection{Sample Preparation}

Methacrylamido propyl trimethyl ammonium chloride (MAPTAC, $50 \mathrm{wt} \%$ in water) was purchased from Sigma Aldrich (Shanghai, China). MAPTAC was purified by using column chromatography and was titrated with $0.1 \mathrm{~mol} / \mathrm{L} \mathrm{AgNO}_{3}$ solution to determine the concentration. 2-acrylamido-2-methyl propane sulfonic acid (AMPS) was provided by Sinopharmacy Corporation (Shanghai, China) and was purified by recrystallization in $\mathrm{CH}_{3} \mathrm{OH}$ twice before use. Azodiisobutyronitrile (AIBN) was purchased from Chengdu Kelong Chemical Corporation (Chengdu, China) and was purified by recrystallization in $\mathrm{CH}_{3} \mathrm{CH}_{2} \mathrm{OH}$ for three times before use. $\mathrm{NaOH}$, $\mathrm{NaCl}, \mathrm{CH}_{3} \mathrm{OH}, \mathrm{CH}_{3} \mathrm{CH}_{2} \mathrm{OH}$ were provided and used without purification by Chengdu Kelong Chemical Corporation. All the chemicals above are of analytic reagent. The Bent used in this paper was purchased from Xinjiang Xiazijie Company (Urumqi, China). The bentonite was purified via a following procedure: Five weight percent Bent and $0.25 \mathrm{wt} \% \mathrm{Na}_{2} \mathrm{CO}_{3}$ were dissolved in deionized water, and the solution was aged at room temperature for $24 \mathrm{~h}$; the mixture was centrifugated at 10,000 RPM to isolate Bent; the Bent was dried under $110^{\circ} \mathrm{C}$, and then sifted with a mesh \#100. The purified Bent was tested according to the China Industrial Standard GB/T20973-2007, the cation exchange capacity (CEC) was $85.8 \mathrm{cmol} \cdot \mathrm{kg}^{-1}$, and the swelling volume was $38.5 \mathrm{~mL} \cdot \mathrm{g}^{-1}$.

The synthesis of 2-[Dodecylthio(thiocarbonyl)thio]-2-methylpropionic acid (DDMAT) was conducted according to a previous related paper [25], and the molecular structure of DDMAT was characterized by ${ }^{1} \mathrm{H}$ NMR, as shown in Figure 1 .

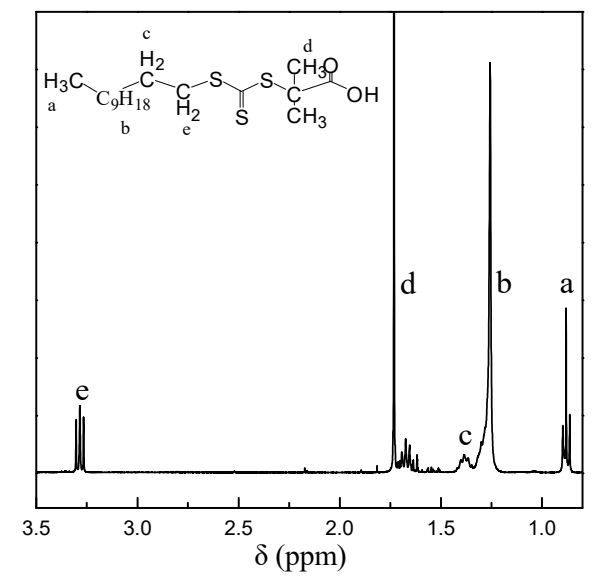

Figure 1. The ${ }^{1} \mathrm{H}$ NMR spectrum of 2-[Dodecylthio(thiocarbonyl)thio]-2-methylpropionic acid (DDMAT). 
The block polyampholyte PAMPS-b-PMAPTAC (Pblock) was produced via a two-step reaction. This first step was to synthesize a macro-chain transfer agent, Macro-PAMPS (Figure 2), and the second step was to synthesize Pblock via a RAFT polymerization between Macro-PAMPS and MAPTAC monomers (Figure 3).

Macro-PAMPS was synthesized according to the following procedure: $\mathrm{CH}_{3} \mathrm{OH}$ and deionized water (3:1 in volume ratio) were added into a flask in icy water, and the solution was purged with Ar for $30 \mathrm{~min}$. AMPS monomers were added to the solution, and then $\mathrm{NaOH}$ was used to adjust the $\mathrm{pH}$ of the solution to around neutral. The flask was distilled to vacuum, and was purged with Ar for $10 \mathrm{~min}$, which was repeated for three times. DDMAT and azobisisobutyronitrile (AIBN), dissolved in $\mathrm{CH}_{3} \mathrm{OH} / \mathrm{H}_{2} \mathrm{O}$, were successively added to the solution via injection. The flask was sealed and heated to $65{ }^{\circ} \mathrm{C}$, and the reaction lasted for $7 \mathrm{~h}$. When the polymerization was completed, the product was transferred to a dialysis bag ( $3500 \mathrm{D})$ in deionized water for $24 \mathrm{~h}$. The dialysis treatment was repeated for three times. Then the product was purified using the freeze-drying technique.

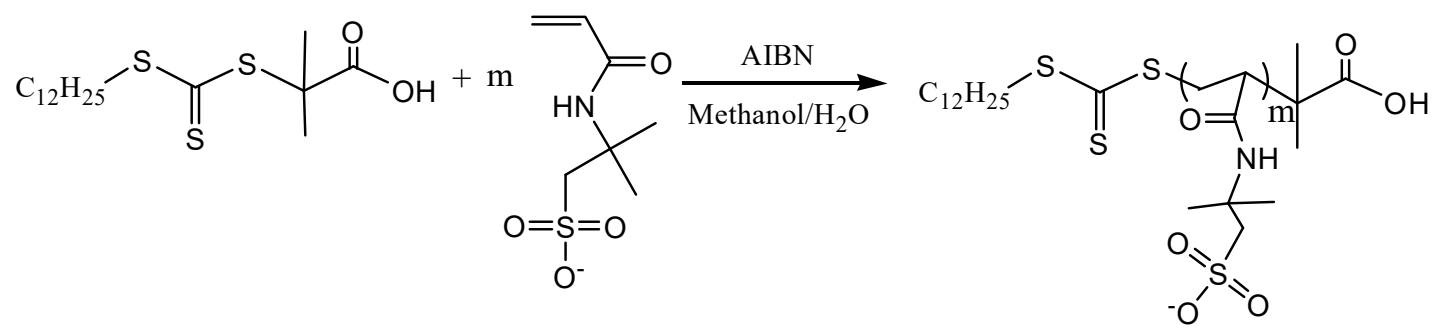

Figure 2. The synthesis route of Macro-PAMPS (first step).

The preparation of Pblock was specified as follows: a certain amount of PAMPS-DDMAT was dissolved in $\mathrm{CH}_{3} \mathrm{OH}$ and deionized water (3:1 in volume ratio), and MAPTAC was added later. After all materials were dissolved, the flask containing the solution was distilled to vacuum and injected with Ar for $10 \mathrm{~min}$, which was repeated for three times. AIBN solution was added to the solution via injection. The flask was sealed and was heated to $65^{\circ} \mathrm{C}$. After reacting for $6 \mathrm{~h}$, the product in the flask was distilled with $\mathrm{CH}_{3} \mathrm{CH}_{2} \mathrm{OH}$ four times. Then the product was transferred to the vacuum oven and dried at $50{ }^{\circ} \mathrm{C}$ for $24 \mathrm{~h}$.
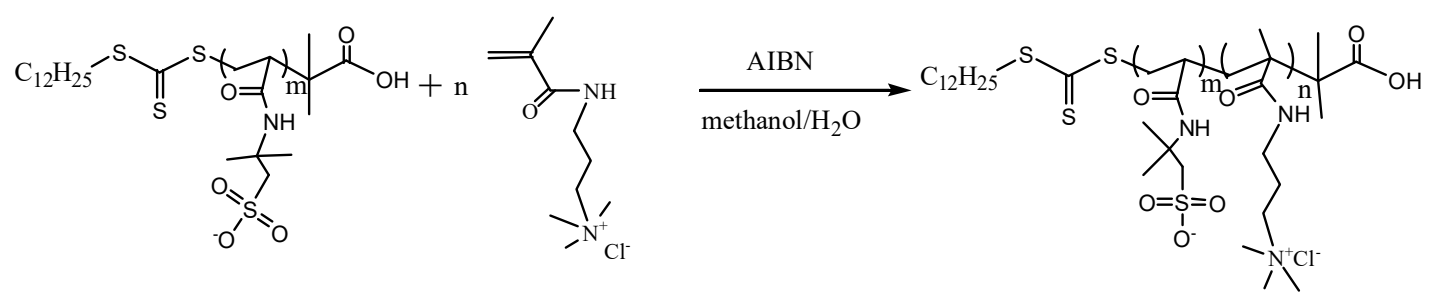

Figure 3. The synthesis route of PAMPS-b-PMAPTAC (second step).

The random polyampholyte PAMPS-s-PMAPTAC (Prandom) was prepared using one-step reaction. AMPS monomers and MAPTAC monomers were dissolved in the mixture of $\mathrm{CH}_{3} \mathrm{OH}$ and deionized water (3:1 in volume ratio). After removal of dissolved $\mathrm{O}_{2}$ in the solution by injecting Ar for $10 \mathrm{~min}$, another solution containing both AIBN and DDMAT was injected into the monomer solution. The following procedure was the same as that of Pblock.

\subsection{FTIR}

The infrared spectra of polymers were tested using a WQF-520 FT-IR device (scans: 16, resolution: $0.5 \mathrm{~cm}^{-1}$ ) from Beijing Rayleigh Analytical Instrument Corporation, Beijing, China. 


\section{3. ${ }^{1} H N M R$}

The ${ }^{1} \mathrm{H}$ NMR spectra were obtained using a Bruker Ascend ${ }^{\mathrm{TM}} 400 \mathrm{MHz}$ NMR spectrometer $\left(\mathrm{D}_{2} \mathrm{O}\right.$ as solvent, narrow bore, static, relaxation time was $2 \mathrm{~s}$, Bruker Company, Billerica, MA, USA).

\subsection{GPC}

The molecular weight distribution of polymers was tested with Waters e2695 GPC (solvent: $1 \mathrm{~mol} / \mathrm{L} \mathrm{NaNO}_{3}, 0.5 \mathrm{~mL} / \mathrm{min}, 35^{\circ} \mathrm{C}$, polyacrylamide of narrow distribution as the standard substance, Waters Company, Milford, MA, USA).

\subsection{SEM}

The microstructure of polymers in aqueous solutions was observed using an FEI QUANTA450 (Thermo Fisher Scientific, Hillsboro, OR, USA) scanning electron microscope (SEM), with a pretreatment including quick-freeze of the solutions, sublimation of $\mathrm{H}_{2} \mathrm{O}$, and gold spraying in the surface of the samples.

\subsection{Zeta Potential}

The zeta potential of polymer solutions was tested using a Brookhaven ZetaPALS instrument (Brookhaven Instrument Corporation, Long Island, NY, USA). Five replications were conducted in each zeta potential test.

\section{7. $X R D$}

The X-ray diffraction (XRD) tests were performed using X'PERT PRO MPD X-ray diffractometer (Malvern Panalytical, Almelo, The Netherlands), operated at $40 \mathrm{kV}$ and $150 \mathrm{~mA}$ with $\mathrm{Cu}-\mathrm{K} \alpha$ radiation $(\lambda=0.154056 \mathrm{~nm})$ at a scanning speed of $0.02^{\circ} / \mathrm{s}$ from $3^{\circ}$ to $20^{\circ}$.

\subsection{Adsorption Tests}

The adsorption test was carried out as follows: First, $5 \mathrm{wt} \%$ purified Bent was dissolved in deionized water, and the solution was stirred for $24 \mathrm{~h}$ under a certain temperature; $5 \mathrm{wt} \%$ polymer solution was prepared in the same way. Then the two solutions were mixed together with a volume ratio of 2:1, and $\mathrm{NaCl}$ of 0 to $30 \mathrm{wt} \%$ was added. Third, the mixture was stirred for 0.1 to $24 \mathrm{~h}$ under 35 to $65^{\circ} \mathrm{C}$. Forth, the mixture was centrifugated at 10,000 RPM to separate the Bent with adsorbed polymers at the bottom of a centrifuge tube. Fifth, the content of polymers in Bent was calculated based on the content of carbon in Bent, using an apparatus of element analysis, Elementar Vario EL-III (Elementar Company, Langenselbold, Hesse, Germany). The content of carbon in purified Bent was tested and the result was $0 \mathrm{wt} \%$.

\section{Results and Discussion}

\subsection{Characterization}

The molecular weight of synthesized Macro-PAMPS in the first step (Figure 2), was characterized by GPC, as shown in Figure 4.

The conversion rate of AMPS monomers increased as the reaction elongated, accompanied by a shift of the eluted peak towards the left, indicating that the molecular weight of the Macro-PAMPS increased as the monomer conversion enhanced. Mn and the monomer conversion appeared to be a linear relationship, showing that the chain propagation of AMPS monomers was well controlled in the first step using the RAFT method. The polydispersity index (PDI) stayed at around 1.25 after the conversion increased to over 30\%. All the data indicated that Macro-PAMPS with a narrow PDI was successfully synthesized. 

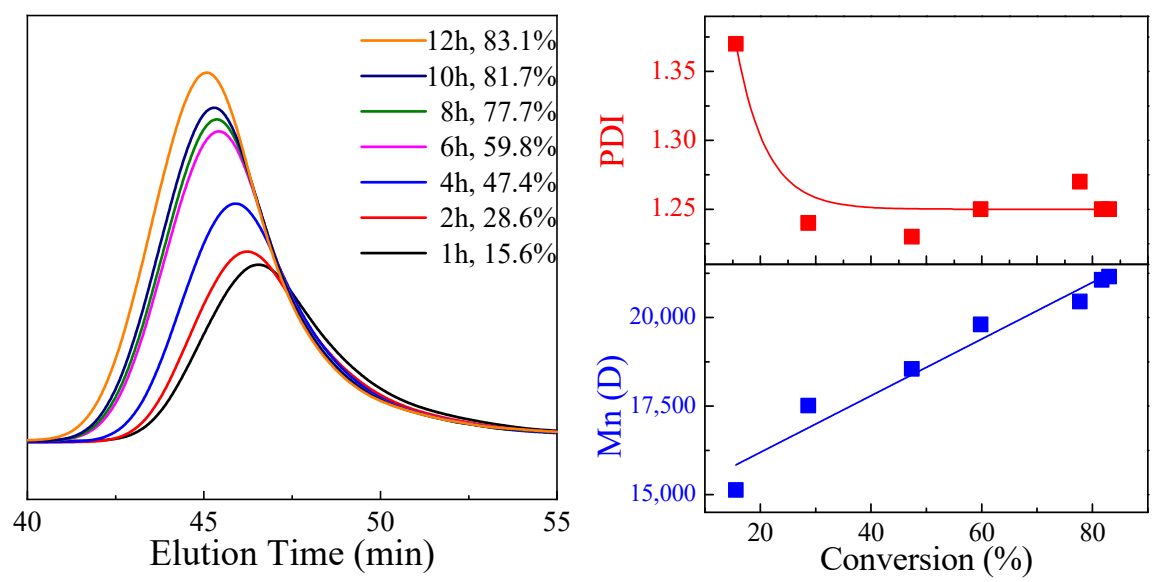

Figure 4. The Gel Permeation Chromatography (GPC) data of Macro-PAMPS prepared in the first step.

The products of the second step, Pblock, were also characterized using GPC, as shown in Table 1 and Figure 5. As the addition of MAPTAC monomers increased, the ratio of MAPTAC units to AMPS units in Pblock increased along with a growing molecular weight of Pblock, supported by the shift of elution peaks towards the left (Figure 5). PDI of Pblock also grew larger but was still under control and less than 1.5 .

Table 1. The molecular parameters of Macro-PAMPS prepared in the first step and block polyampholyte synthesized in the second step.

\begin{tabular}{cccc}
\hline Sample & $\mathbf{n}_{\text {AMPS }}: \mathbf{n}_{\text {MAPTAC }}$ & Mn (D) & PDI \\
\hline Macro-PAMPS & - & 10,500 & 1.21 \\
Pblock-1 & $4.35: 1$ & 12,600 & 1.25 \\
Pblock-2 & $2.13: 1$ & 14,300 & 1.28 \\
Pblock-3 & $0.85: 1$ & 16,600 & 1.35 \\
\hline
\end{tabular}

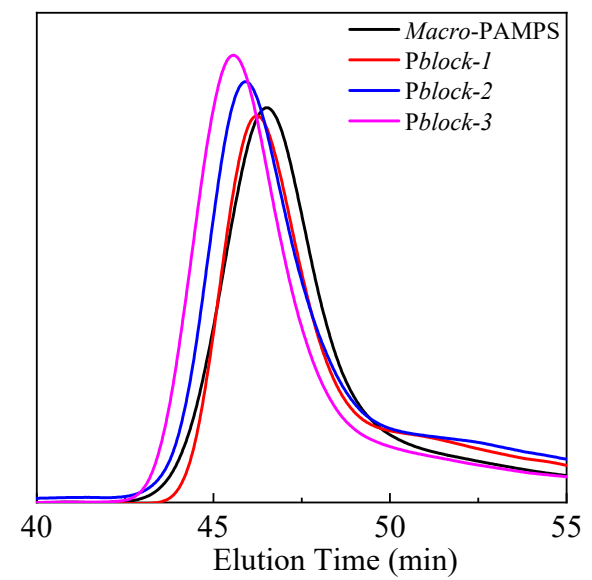

Figure 5. The elution peaks of Macro-PAMPS prepared in the first step and block polyampholyte synthesized in the second step.

The PDI of Pblock and that of Macro-PAMPS were almost the same, demonstrating that block polyampholyte with narrow molecular weight distribution was successfully prepared.

Next, the molecular structure of polymers was characterized by FTIR and ${ }^{1} \mathrm{H}$ NMR (Figure 6). 

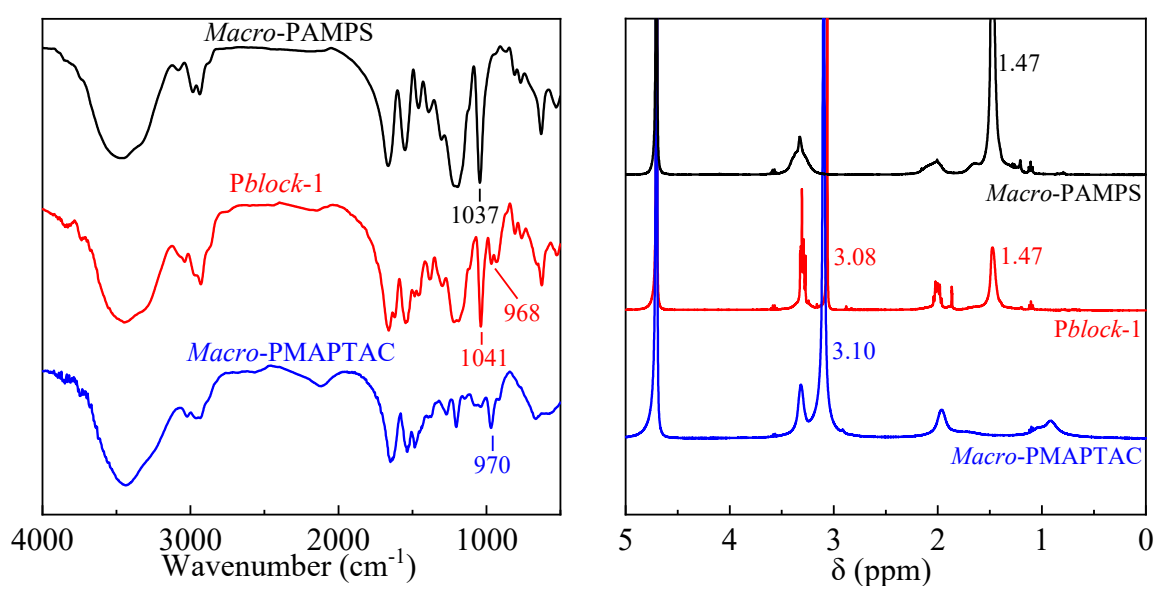

Figure 6. FTIR and ${ }^{1} \mathrm{H}$ NMR of Macro-PAMPS, Pblock-1, Macro-PMAPTAC.

The band around $1041 \mathrm{~cm}^{-1}$ corresponded to the symmetric stretching vibration of $-\mathrm{SO}^{3-}$ in AMPS unit, and the signal around $970 \mathrm{~cm}^{-1}$ was attributed to the stretching vibration of quaternary ammonium group in MAPTAC moieties. The peak at $1.47 \mathrm{ppm}$ was introduced by the $-\mathrm{CH}_{3}$ in AMPS unit, while the peak around $3.10 \mathrm{ppm}$ corresponded to the quaternary ammonium group in MAPTAC moieties. The results demonstrated that the target Pblock have been successfully synthesized via two-step reaction using the RAFT method.

A batch of polymers carrying net negative charges including Macro-PAMPS, block polyampholyte and random polyampholyte, as shown in Table 2, were synthesized using the RAFT methods for the adsorption test by tuning the feed ratio of AMPS monomers and MAPTAC monomers.

Table 2. The molecular parameters of polymer samples used in the following adsorption tests.

\begin{tabular}{cccc}
\hline Sample & $\mathbf{n}_{\text {AMPS }}: \mathbf{n}_{\text {MAPTAC }}$ & Mn (D) & PDI \\
\hline Macro-PAMPS & - & 28,600 & 1.23 \\
Pblock-4 & $5.99: 1$ & 24,500 & 1.30 \\
Pblock-5 & $3.99: 1$ & 24,700 & 1.39 \\
Pblock-6 & $4.01: 1$ & 26,700 & 1.35 \\
Prandom & $3.56: 1$ & 21,800 & 1.41 \\
\hline
\end{tabular}

Considering that quaternary ammonium groups and sulfonate groups are stable in a wide range of $\mathrm{pH}$ under room temperature, the polymers in Table 2 have little sensitivity to the $\mathrm{pH}$ variation of the solutions. As the following adsorption tests were carried out at neutral $\mathrm{pH}$, the precipitation of both random polyampholyte and block polyampholyte cannot be observed as their isoelectric point (IEP) point region exists at $\mathrm{pH}<2$.

\subsection{The Microstructure of Polyampholyte Solutions}

The microstructure of polymers (Table 2$)$ before $(0.05 \mathrm{wt} \%$ polymer $)$ and after $(0.05 \mathrm{wt} \%$ polymer $+0.05 \mathrm{wt} \% \mathrm{NaCl}$ ) the intrusion of salt was studied using SEM, and the results are shown as Figure 7. 

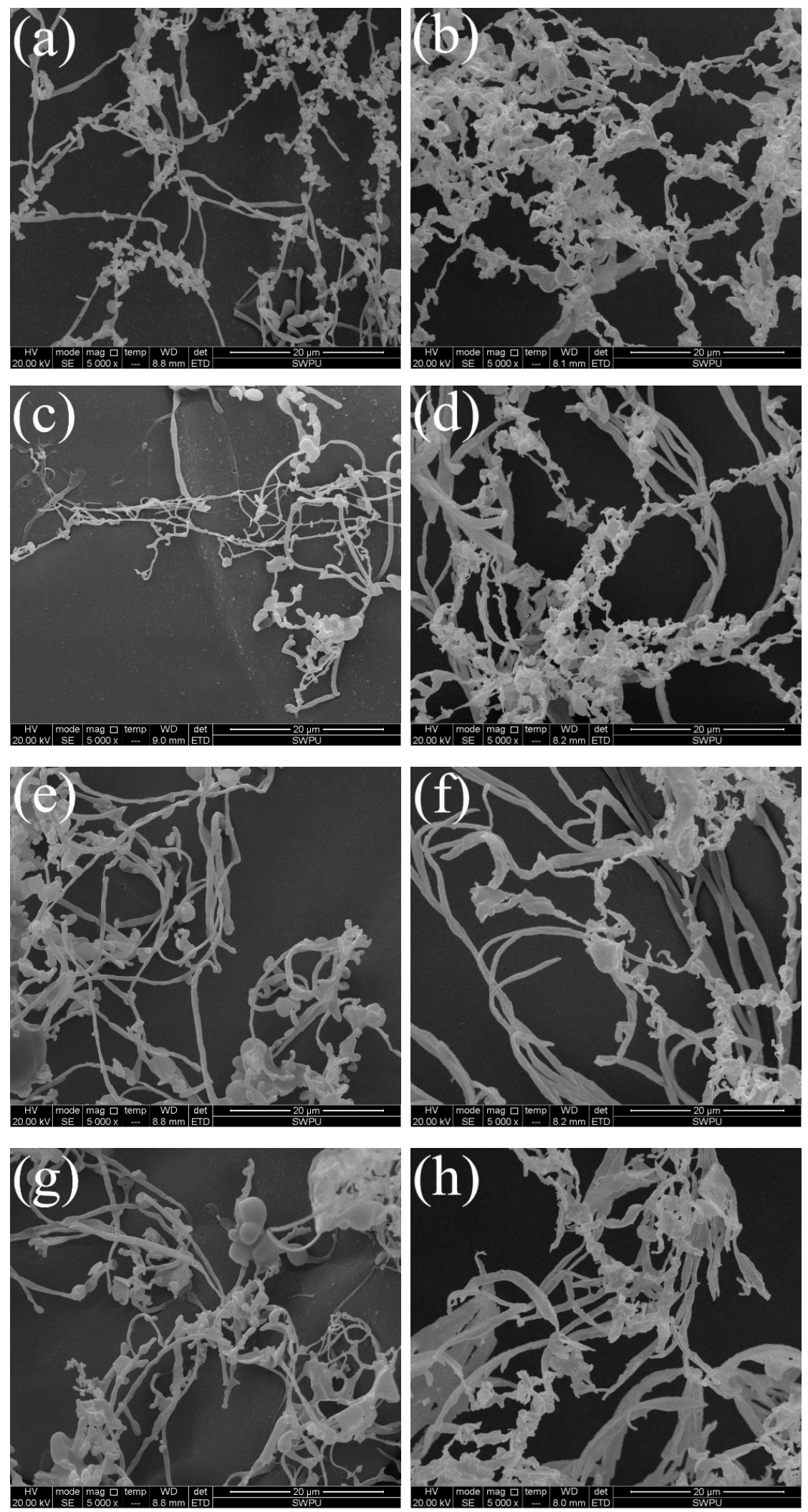

Figure 7. Cont. 

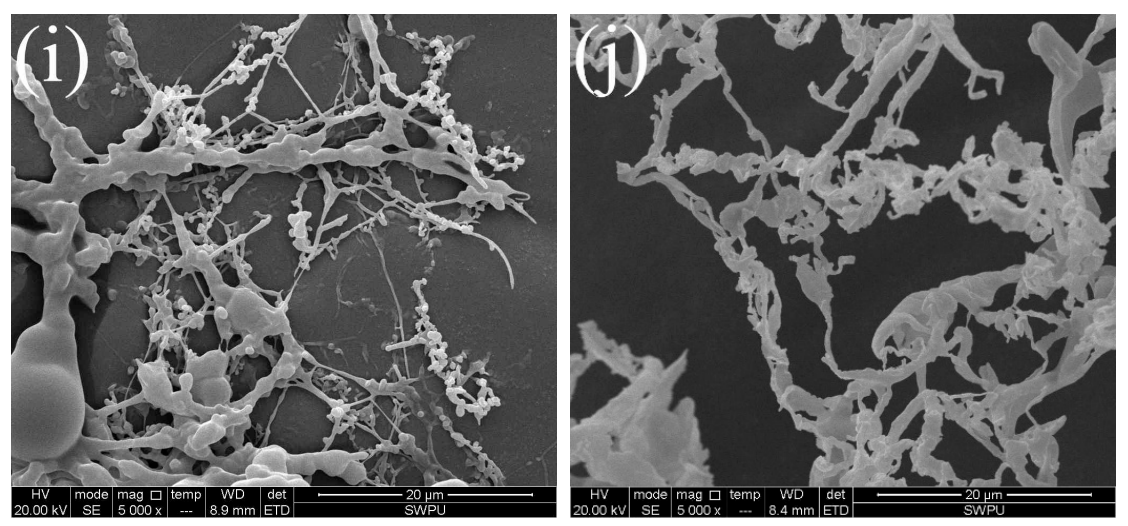

Figure 7. The microstructure of Macro-PAMPS, Pblock-4, Pblock-5, Pblock-6, and Prandom in the absence $(\mathbf{a}, \mathbf{c}, \mathbf{e}, \mathbf{g}, \mathbf{i})$ and presence $(\mathbf{b}, \mathbf{d}, \mathbf{f}, \mathbf{h}, \mathbf{j})$ of $\mathrm{NaCl}$.

The chains of Prandom extended upon the intrusion of salt. In deionized water, the chains of random polyampholyte were inclined to be in globular conformation, driven by the intensive electrostatic attraction between adjacent oppositely charged groups. The intrusion of $\mathrm{Na}^{+}$along with the solvent into the space among the entangled chains resulted in a screening of the electrostatic attraction. Therefore, the condensed chains tended to expand in a disordered way.

Both Pblock samples and Macro-PAMPS suffered less from the addition of $\mathrm{NaCl}$. The oppositely charged groups of Pblock samples were partially distributed along the chain, and their electrostatic interaction exerted less effect on the microstructure compared with that of Pblock.

The zeta potential of polymers (Table 3) in the presence and absence of $\mathrm{NaCl}$ was in agreement with the molecular parameters of these samples. Macro-PAMPS had the lowest zeta potential. As more MAPTAC units were introduced into Pblock, the zeta potential began to increase. The molecular weight also influenced the zeta potential, as Pblock-6 had a lower zeta potential than Pblock-5. After the addition of salt, the zeta potential of all samples suffered greatly, as $\mathrm{Na}^{+}$considerably compressed the hydration layer of charged groups.

Table 3. The zeta potential of polymer solutions.

\begin{tabular}{ccc}
\hline Sample & Deionized Water & Brine Water \\
\hline Macro-PAMPS & -93.44 & -49.14 \\
Pblock-4 & -78.57 & -36.94 \\
Pblock-5 & -40.68 & -13.48 \\
Pblock-6 & -50.59 & -18.68 \\
Prandom & -38.30 & -12.43 \\
\hline
\end{tabular}

\subsection{The Adsorptive Behavior of Polyampholytes}

The adsorption tests were conducted in aqueous solutions, with stirring time, temperature, and salinity as the external factors, as shown in Figure 8. 

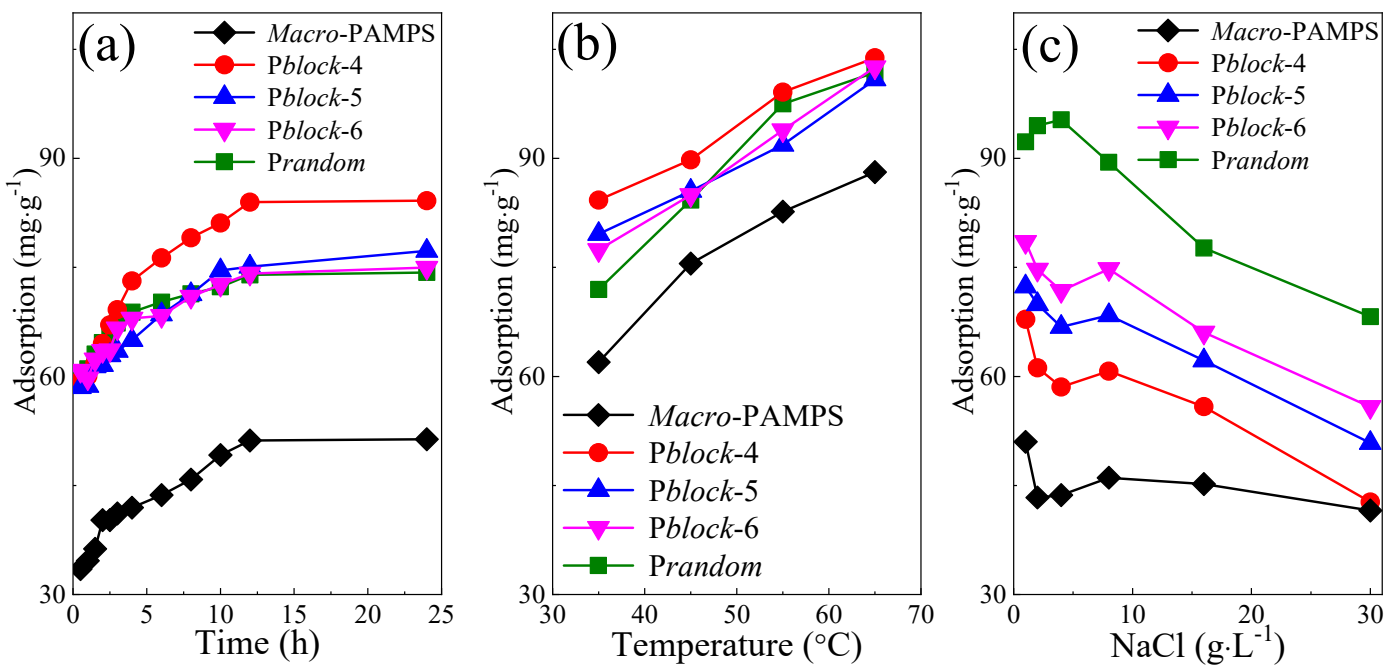

Figure 8. The effect of time (a), temperature (b), salt (c) on the adsorption of Macro-PAMPS, block polyampholyte and random polyampholyte.

The experiments on adsorption kinetics were carried out in deionized water at $30^{\circ} \mathrm{C}$. In Figure $8 \mathrm{a}$, it seemed that no distinct difference among the adsorption of block polyampholyte and that of random polyampholyte, except that Pblock-4 exhibited a stronger affinity to Bent. Macro-PAMPS had much lower adsorption than polyampholyte, as the former only carries polar groups and anionic groups which had lower adsorptive ability than cationic groups. Several points should be emphasized here. The first point is that even an intense interaction between cationic groups and anionic groups in random polyampholyte and block polyampholyte existed according to the observed microstructure (Figure 7), cationic groups in the chains still provided the polyampholyte with more potential adsorption sites than polar groups did in the case of polyanion. The second point is that even if the adsorbent and the adsorbate carry the same type of charge, adsorption can still occur between them. The third point is that the adsorption equilibrium of all polymers in the adsorption experiments was reached after $12 \mathrm{~h}$.

The influence of heating on the adsorption (the Bent-polymer solution was mixed for $12 \mathrm{~h}$ ) of polymers was also studied in deionized water. The adsorption of block polyampholyte and random polyampholyte was almost the same, according to Figure $8 \mathrm{~b}$. An interesting phenomenon was observed that the adsorption of Macro-PAMPS and polyampholyte were promoted by the increase of temperature in the range of 35 to $65^{\circ} \mathrm{C}$. The first possible explanation is that the pronounced thermal motion induced by increased temperature weakened the electrostatic binding between oppositely charged groups, and cationic groups getting rid of the electrostatic bound adsorbed on Bent's negatively charged layer surface. The second explanation is that a partial expansion of the macromolecular chains, as a consequence of the enhancement of the solvent quality, may also favor the escape of cationic groups from the entangled or even condensed polymer segments [26].

The adsorption tests (the Bent-polymer solution was mixed for $12 \mathrm{~h}$ ) in the presence of $\mathrm{NaCl}$ were carried out at $30^{\circ} \mathrm{C}$. In Figure 8c, the salt exerted a negative effect on the adsorption of all polymers. $\mathrm{As} \mathrm{Na}^{+}$permeated into the space among charged groups and compressed the double electric layer of them, the entangled or even condensed chains via electrostatic attraction tended to stay away from one another. The inter-chain entanglement driven by hydrogen bond also suffered significantly from the intrusion of ions. On the other hand, the double electric layer of Bent was also compressed by ionized $\mathrm{NaCl}$, and a considerable amount of $\mathrm{Na}^{+}$surrounded the interlayer surface of Bent. Therefore, the interactions between Bent and charged groups or polar groups became less effective, leading to less adsorption of polymers.

Pblock-6, sharing a similar monomer ratio with Pblock-5 but a higher Mn than the later, displayed higher adsorption than Pblock-5. This result may reveal that the average length of the chain mainly determines both the number of potential adsorptive groups and the adsorption of the polymer. 
It is also worth mentioning that Prandom suffered less from the increased salinity than Pblock samples. Considering that the former exhibited less adsorption than the later in the absence of $\mathrm{NaCl}$, we can infer that molecular weight contributed more to the adsorption of polyampholyte in deionized water, while the content of cationic group dominated the adsorption of polyampholyte in the presence of salt, as Prandom had a higher percentage of cationic moieties but lower Mn compared with Pblock (Table 2). Macro-AMPS still had the lowest adsorption after the intrusion of salt, stemming from its lowest zeta potential (Table 3).

The structure of Bent before and after the adsorption of polymers was characterized by XRD. Figure 9a illustrated the composition of Bent. Ill/Sm, Ill, Kaol and Qtz refer to Illite/Smectite, Illite, Kaolinte and Quartz, respectively. Polymers mainly adsorbed on Illite/Smectite. According to Figure $9 b$, the $d_{001}$ basal spacing $\left(2 \theta \approx 7^{\circ}\right)$ of Bent was weakened and moved towards a small degree as different polymers adsorbed in flat layer surface [27]. Assuming pole conformation, fence conformation or pancake conformation, the polymer chains enclosed Bent plates, and increased both the electrostatic repulsion and entropic repulsion among those particles, resulting in a disordered distribution of Bent lattice planes. More importantly, the interlayer spacing of Bent lattice planes grew larger as polymers intercalated into the interlayer space in Bent particles, as the $\mathrm{d}_{001}$ basal spacing of Pblock-4/Bent, Pblock-5/Bent, and Pblock-6/Bent moved towards a smaller angle compared with purified Bent. This result indicated that the polymers adsorbed on the flat layer surface of Bent particles. The cationic groups in Pblock were distributed as a segment, interacting with the negatively charged interlayer surface of Bent via a multi-point adsorption.

The $\mathrm{d}_{001}$ basal spacing of Macro-PAMPS/Bent and Prandom/Bent had rarely shifted, which means that the adsorption of these polymers exhibited less effect on the expansion of Bent interlayer space. The statistical distribution of positive charged groups in Prandom had an adverse effect on the interaction between these cationic groups and the negatively charged interlayer surface of Bent, resulting in both lower $\mathrm{d}_{001}$ basal spacing of Prandom/Bent and less adsorption.
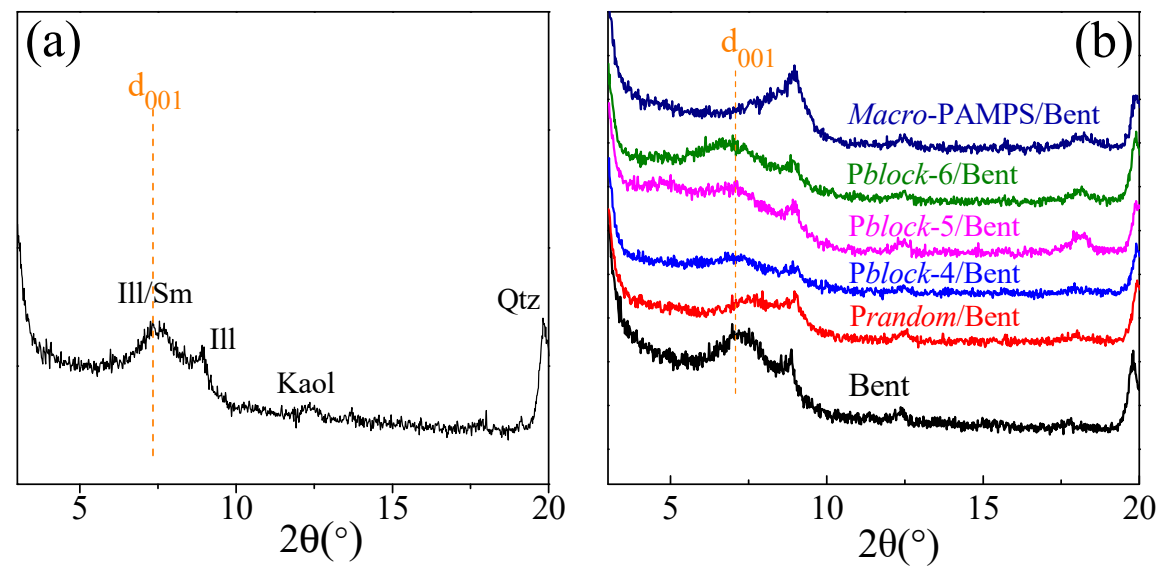

Figure 9. The X-ray diffraction (XRD) analysis of bentonite (Bent) (a) and Bent with adsorbed polymers (b).

As block polyampholyte bearing net negative charges exhibited a greater adsorptive ability on Bent than random polyampholyte according to the analysis of XRD tests and adsorption tests, the former may find a broad application in water-based mud of oil and gas industry and the synthesis of polymer-clay nanocomposites.

\section{Conclusions}

In this paper, we synthesized block polyampholyte using the RAFT method, with AMPS and MAPTAC monomers. A macro transfer agent Macro-PAMPS was prepared, and then the copolymerization 
between Macro-PAMPS and MAPTAC monomers initiated by AIBN was conducted. FTIR, ${ }^{1} \mathrm{H}$ NMR, and GPC results demonstrated that the target block polyampholyte was successfully synthesized.

The partial distribution of groups carrying like charges in polyampholyte led to an intense entanglement of polymer chains, while the random distribution of oppositely charged groups resulted in a condense conformation of the chains. Addition of salt weakened the electrostatic interaction between charged groups and, therefore, influenced the microstructure of polyampholyte. The zeta potential of Macro-PAMPS and polyampholyte decreased considerably upon the intrusion of $\mathrm{NaCl}$.

The adsorption kinetic study revealed that after $12 \mathrm{~h}$, Macro-PAMPS and polyampholyte reached the adsorption equilibrium on Bent. Increasing temperature in a proper range contributed to the adsorption while the introduction of $\mathrm{NaCl}$ affected the adsorption. In deionized water, a better way to increase the adsorption of polyampholyte on Bent is to increase its molecular weight; in the presence of salt, introducing more cationic moieties seems to be a more practical method to enhance the adsorption of polyampholyte.

Block polyampholyte had a stronger adsorptive affinity to Bent than random polyampholyte in the absence of salt, and the former showed higher intercalation ability in enlarging the $\mathrm{d}_{001}$ basal spacing of Bent.

Author Contributions: Funding acquisition, L.L.; Investigation, Y.L. and X.L.; Methodology, L.L. and Y.L.; Projection administration, L.L.; Supervision, L.L.; Validation, L.L.; Writing-original draft, Y.L.; Writing-review and editing, L.L.

Funding: This research was funded by the National Natural Science Foundation of China (41702391) and the Young Scholars Development Fund of SWPU (201599010037).

Acknowledgments: The authors wish to acknowledge Pingya Luo, Southwest Petroleum University, and Qi Chu, Sinopec Research Institute of Petroleum Engineering, for their advice on experimental design.

Conflicts of Interest: The authors declare no conflict of interest.

\section{References}

1. Dobrynin, A.V.; Colby, R.H.; Rubinstein, M. Polyampholytes. J. Polym. Sci. Part B Polym. Phys. 2004, 42, 3513-3538. [CrossRef]

2. Chu, Q.; Lin, L. Synthesis and properties of an improved agent with restricted viscosity and shearing strength in water-based drilling fluid. J. Pet. Sci. Eng. 2019, 173, 1254-1263. [CrossRef]

3. Lin, L.; Luo, P. Amphoteric hydrolyzed poly(acrylamide/dimethyl diallyl ammonium chloride) as a filtration reducer under high temperatures and high salinities. J. Appl. Polym. Sci. 2015, 132, 41581. [CrossRef]

4. Pafiti, K.S.; Philippou, Z.; Loizou, E.; Porcar, L.; Patrickios, C.S. End-Linked poly[2-(dimethylamino)ethyl methacrylate]-poly(methacrylic acid) polyampholyte conetworks: Synthesis by sequential RAFT polymerization and swelling and SANS characterization. Macromolecules 2011, 44, 5352-5362. [CrossRef]

5. Stavrouli, N.; Aubry, T.; Tsitsilianis, C. Rheological properties of ABA telechelic polyelectrolyte and ABA polyampholyte reversible hydrogels: A comparative study. Polymer 2008, 49, 1249-1256. [CrossRef]

6. You, J.; Hu, H.; Zhou, J. Synthesis, structure and solution properties of the novel polyampholytes based on cellulose. Cellulose 2013, 20, 1175-1185. [CrossRef]

7. Mahltig, B.; Je' rôme, R.; Stamm, M. Diblock polyampholytes at the silicon/water interface: Adsorption at various modified silicon substrates. Phys. Chem. Chem. Phys. 2001, 3, 4371-4375. [CrossRef]

8. Bekturov, E.A.; Kudaibergenov, S.E.; Khamzamulina, R.E.; Frolova, V.A.; Nurgalieva, D.E. Phase behaviour of block-polyampholytes based on poly(methacrylic acid)-block-poly(1-methyl-4-vinylpyridinium chloride) in aqueous salt solutions. Macromol. Rapid Commun. 1992, 13, 225-229. [CrossRef]

9. Sfika, V.; Tsitsilianis, C. Association phenomena of poly(acrylic acid)-b-poly(2-vinylpyridine)-b-poly(acrylic acid) triblock polyampholyte in aqueous solutions from transient network to compact micelles. Macromolecules 2003, 36, 4983-4988. [CrossRef]

10. Dyakonova, M.A.; Stavrouli, N.; Popescu, M.T.; Kyriakos, K.; Grillo, I.; Philipp, M.; Jaksch, S.; Tsitsilianis, C.; Papadakis, C.M. Physical hydrogels via charge driven self-organization of a triblock polyampholyte-Rheological and structural investigations. Macromolecules 2014, 47, 7561-7572. [CrossRef] 
11. Dyakonova, M.A.; Berezkin, A.V.; Kyriakos, K.; Gkermpoura, S.; Popescu, M.T.; Filippov, S.K.; Štěpánek, P.; Di, Z.; Tsitsilianis, C.; Papadakis, C.M. Salt-Induced changes in triblock polyampholyte hydrogels: Computer simulations and rheological, structural, and dynamic characterization. Macromolecules 2015, 48, 8177-8189.

12. Tsitsilianis, C.; Stavrouli, N.; Bocharova, V.; Angelopoulos, S.; Kiriy, A.; Katsampas, I.; Stamm, M. Stimuli responsive associative polyampholytes based on ABCBA pentablock terpolymer architecture. Polymer 2008, 49, 2996-3006. [CrossRef]

13. Yu, K.; Han, Y. Effect of block sequence and block length on the stimuli-responsive behavior of polyampholyte brushes: Hydrogen bonding and electrostatic interaction as the driving force for surface rearrangement. Soft Matter 2009, 5, 759-768. [CrossRef]

14. Hammond, M.R.; Li, C.; Tsitsilianis, C.; Mezzenga, R. Hierarchical self-organization in polyelectrolyte-surfactant complexes based on heteroarm star block copolyampholytes. Soft Matter 2009, 5, 2371. [CrossRef]

15. Stavrouli, N.; Katsampas, I.; Aggelopoulos, S.; Tsitsilianis, C. pH/Thermosensitive hydrogels formed at low pH by a PMMA-PAA-P2VP-PAA-PMMA pentablock terpolymer. Macromol. Rapid Commun. 2008, 29, 130-135. [CrossRef]

16. Zurick, K.M.; Bernards, M. Recent biomedical advances with polyampholyte polymers. J. Appl. Polym. Sci. 2014, 131, 596-602. [CrossRef]

17. Copello, G.J.; Diaz, L.E.; Dall'Orto, V.C. Adsorption of $\mathrm{Cd}(\mathrm{II})$ and $\mathrm{Pb}(\mathrm{II})$ onto a one step-synthesized polyampholyte: Kinetics and equilibrium studies. J. Hazard. Mater. 2012, 217-218, 374-381. [CrossRef]

18. Mishra, R.K.; Ramasamy, K.; Ban, N.N.; Majeed, A.B.A. Synthesis of poly[3-(methacryloylamino) propyl trimethylammonium chloride-co-methacrylic acid] copolymer hydrogels for controlled indomethacin delivery. J. Appl. Polym. Sci. 2013, 128, 3365-3374. [CrossRef]

19. Dobbins, S.C.; Mcgrath, D.E.; Bernards, M.T. Nonfouling hydrogels formed from charged monomer subunits. J. Phys. Chem. B 2012, 116, 14346-14352. [CrossRef]

20. Chakrabarty, T.; Shahi, V.K. (3-glycidoxypropyl) Trimethoxy silane induced switchable zwitterionic membrane with high protein capture and separation properties. J. Membr. Sci. 2013, 444, 77-86. [CrossRef]

21. Lin, L.; Luo, P. Effect of polyampholyte-bentonite interactions on the properties of saltwater mud. Appl. Clay Sci. 2018, 163, 10-19. [CrossRef]

22. Shaikh, S.M.R.; Nasser, M.S.; Hussein, I.A.; Benamor, A. Investigation of the effect of polyelectrolyte structure and type on the electrokinetics and flocculation behavior of bentonite dispersions. Chem. Eng. J. 2017, 311, 265-276. [CrossRef]

23. Shaikh, S.M.R.; Nasser, M.S.; Magzoub, M.; Benamor, A.; Hussein, I.A.; El-Naas, M.H.; Qiblawey, H. Effect of electrolytes on electrokinetics and flocculation behavior of bentonite-polyacrylamide dispersions. Appl. Clay Sci. 2018, 158, 46-54. [CrossRef]

24. Ahmad, H.M.; Kamal, M.S.; Al-Harthi, M.A. Rheological and filtration properties of clay-polymer systems: Impact of polymer structure. Appl. Clay Sci. 2018, 160, 226-237. [CrossRef]

25. Lai, J.T.; Filla, D.; Shea, R. Functional polymers from novel carboxyl-terminated trithiocarbonates as highly efficient RAFT agents. Macromolecules 2002, 35, 6754-6756. [CrossRef]

26. Bossard, F.; Tsitsilianis, C.; Yannopoulos, S.N.; Petekidis, G.; Sfika, V. A novel thermothickening phenomenon exhibited by a triblock polyampholyte in aqueous salt-free solutions. Macromolecules 2005, 38, 2883-2888. [CrossRef]

27. Villar, M.V.; Gómez-Espina, R.; Gutiérrez-Nebot, L. Basal spacings of smectite in compacted bentonite. Appl. Clay Sci. 2012, 65-66, 95-105. [CrossRef]

(C) 2018 by the authors. Licensee MDPI, Basel, Switzerland. This article is an open access article distributed under the terms and conditions of the Creative Commons Attribution (CC BY) license (http://creativecommons.org/licenses/by/4.0/). 